\title{
A SUPERFLUID GYROSCOPE WITH COLD ATOMIC GASES
}

\author{
S. Stringari \\ Dipartimento di Fisica, Università di Trento, \\ and Istituto Nazionale per la Fisica della Materia, \\ I-38050 Povo, Italy
}

(November 6, 2018)

\begin{abstract}
A trapped Bose-Einstein condensed atomic gas containing a quantized vortex is predicted to exhibit precession after a sudden rotation of the confining potential. The equations describing the motion of the condensate are derived and the effects of superfluidity explicitly pointed out. The dependence of the precession frequency on the relevant parameters of the problem is discussed. The proposed gyroscope is well suited to explore rotational effects at the level of single quanta of circulation.
\end{abstract}

PACS numbers: 03.75.Fi, 05.30.Jp, 32.80.Pj, 67.40.-w 
After the realization of Bose-Einstein condensation [1] in magnetically trapped vapours of alkali atoms, cooled down to extremely low temperatures, the superfluid behaviour of these systems has become the object of extensive experimental work. This includes the study of rotational properties, like quantized vortices [2,3] and the quenching of the moment of inertia [4], as well as the reduction of dissipative effects [5].

The purpose of this work is to show that Bose-Einstein condensed (BEC) gases can be used to realize a quantum gyroscope where the effects of superfluidity show up in a very peculiar way. Superfluid gyroscopes have been already realized with liquid ${ }^{4} \mathrm{He}[6]$ and ${ }^{3} \mathrm{He}$ [7] and have been mainly used to investigate the nature of persistent currents in toroidal geometries. Most of experiments with helium gyroscopes operate with many quanta of circulation. Compared to liquid helium, trapped BEC gases are mesoscopic systems in the sense that the healing length, which provides a typical range of dynamic correlations and fixes the size of the vortex core, is smaller, but not extremely smaller than the size of the sample [8]. For the same reason the angular momentum $N \hbar$ carried by a single quantized vortex can have visible effects on the global motion of the condensate as we will prove in this letter. A further feature that characterizes these systems is the very peculiar type of confinement which yields new possibilities for exploring superfluid phenomena. Important gyroscopic effects associated with the occurrence of vortex lines have been already observed in trapped BEC gases [9 11]. The authors of [9] have succeeded in testing the quantization of the angular momentum of a single vortex line. To this purpose one generates a quadrupole deformation in the plane orthogonal to the vortex axis. The observed precession of the deformation is proportional to the angular momentum carried by the vortex, in accordance with the predictions of theory [12,13]. Notice that in this experiment the vortex line is not affected by the precession. The authors of [10] and [11] have instead observed the precession of a vortex line either displaced or tilted from the symmetry axis of the condensate. In this case the motion of the condensate is not affected at a macroscopic level and the precession involves the change of the density on a more microscopic scale, of the order of the size of the vortex core. 
In the present work we discuss the macroscopic precession of the symmetry axis of a deformed condensate caused by the sudden rotation of the trap, in the presence of a quantized vortex line. This precession corresponds to a full rotation of the condensate in $3 \mathrm{D}$, which preserves the intrinsic shape of the system.

The geometry of the proposed gyroscope is illustrated in fig.1. The system consists of a dilute and cold gas of atoms with mass $m$ confined by an axi-symmetric trap of harmonic shape:

$$
V_{\text {ext }}=\frac{m}{2}\left(\omega_{\perp}^{2}\left(x^{2}+y^{2}\right)+\omega_{z}^{2} z^{2}\right)
$$

where $\omega_{\perp}$ and $\omega_{z}$ are, respectively, the radial and axial trapping frequencies. The trapping potential is characterized by the deformation parameter

$$
\epsilon=\frac{\left(\omega_{\perp}^{2}-\omega_{z}^{2}\right)}{\left(\omega_{\perp}^{2}+\omega_{z}^{2}\right)}
$$

which will be taken different from zero. As a consequence of this anisotropy the atomic cloud is also deformed. If the atoms are bosons, at very low temperatures they undergo a phase transition associated with the macroscopic occupation of a single-particle state (Bose-Einstein condensation). This happens for temperatures below the critical value $k T_{c}=$ $0.94 \hbar \omega_{h o} N^{1 / 3}$ where $\omega_{h o}=\left(\omega_{z} \omega_{\perp}^{2}\right)^{1 / 3}$ is the geometrical average of the trapping frequencies and $N$ is the number of atoms. Typical values of $T_{c}$ range between 0.1 and $1 \mu K$. Below $T_{c}$ the system exhibits unique features which have been the object of systematic experimental and theoretical investigation. For our purposes the system should be very cold in order to minimize the dissipative effects produced by the thermal cloud which tend to destabilize the vortex line [15, 16]. For this reason we will discuss here the behaviour of the gas at zero temperature.

Let us consider a Bose-Einstein condensate in equilibrium in the trap, with no angular momentum, and let us suppose that the symmetry axis of the trapping potential is suddenly rotated in the $x z$ plane through an angle $\theta_{0}$. After the rotation the gas is no longer in equilibrium and starts oscillating around the new symmetry axis. If the angle of rotation 
is small the gas will keep its intrinsic shape and the motion will correspond to a periodic oscillation characterized by the angle $\theta(t)$, giving rise to the so-called scissors mode [14]. The motion is very different from the one of a classical body because the inertia is strongly suppressed by superfluidity. In particular the oscillation frequency does not vanish when the deformation (2) of the trap becomes small, as one would expect for a classical system.

The calculation of the frequency of the scissors mode requires the solution of a non trivial many-body problem where the interactions among particles and the effects of quantum statistics play an important role. An exact solution of the problem can be obtained for large samples, such that the dimensionless parameter $\mathrm{Na} / \mathrm{a}_{h o}$ is much larger than unity. This limit corresponds to the so-called Thomas-Fermi (TF) regime and is well achieved in many available experimental configurations. Here $a$ is the s-wave scattering length (of the order of $\left.10^{-2}-10^{-3} \mu m\right)$ while $a_{h o}=\sqrt{\hbar /\left(m \omega_{h o}\right)}$ is the oscillator length fixed by the geometrical average of the trapping frequencies (of the order of a few microns in standard magnetic traps). In the TF limit, the equilibrium density takes the form of an inverted parabola: $n(\mathbf{r})=m\left[\mu-V_{\text {ext }}(\mathbf{r})\right] /\left(4 \pi \hbar^{2} a\right)$ for $\mu \geq V_{\text {ext }}$ and zero elsewhere, where $\mu=$ $\left(\omega_{h o} / 2\right)\left(15 N a / a_{h o}\right)^{2 / 5}$ is the chemical potential fixed by the normalization of the density. The surface where the density vanishes defines an ellipsoid whose principal radii $R_{\perp}$ and $R_{z}$ are determined by the relationship $\omega_{\perp}^{2} R_{\perp}^{2}=\omega_{z}^{2} R_{z}^{2}=2 \mu / m$. In the Thomas-Fermi limit the equations of motion take the simplified form of the hydrodynamic theory of superfluids [17]. With respect to the equations of classical hydrodynamics they are characterized but the additional constraint of irrotationality. It is worth noticing that the "hydrodynamic" form of these equations is not the result of collisional processes as happens in classical gases, but is the consequence of superfluidity. These equations admit an analytic solution for the scissors mode with frequency [14]

$$
\omega_{\text {scissors }}=\sqrt{\omega_{\perp}^{2}+\omega_{z}^{2}}
$$

This mode has recently been observed [4] confirming the predictions of theory with high accuracy. 
Let us discuss now the case in which the condensate has initially an intrinsic angular momentum. This can be due to a quantized vortex aligned along the symmetry axis of the condensate, carrying one unit $\hbar$ of angular momentum per particle. The size of the vortex core is fixed by the healing length $\xi=\left(2 m \mu / \hbar^{2}\right)^{-1 / 2}$ and is always smaller than the size of the condensate. Also in the presence of the vortex the sudden rotation of the trap will excite the scissors mode, but now the oscillation will precess due to the torque

$$
\frac{d}{d t}<\mathbf{L}>=\frac{1}{i \hbar}<[\mathbf{L}, H]>=-m<\mathbf{r} \times \nabla V_{e x t}>
$$

produced by the external anisotropic potential (1). Here $\mathbf{L}$ is the angular momentum operator and the expectation value is taken on the quantum mechanical state of the system. The value of the torque along the $y$ axis is $d<L_{y}>/ d t=-m\left(\omega_{\perp}^{2}-\omega_{z}^{2}\right) N<x z>$ and differs from zero immediately after the sudden rotation of the trap in the $x z$-plane, thereby causing the precession.

In order to derive the equation for the rotation of the condensate we notice that, if the angle of rotation $\theta_{0}$ is small compared to the deformation (2) of the trap, the shape of the sample is preserved. Under this condition the whole motion can be described in terms of two angles: the inclination angle $\theta$ and the azimuthal angle $\phi$. This is a remarkable feature because gases are highly compressible and their shape can change very easily. The values of $\theta$ and $\phi$ are related to the averages $\langle x z\rangle$ and $\langle y z\rangle$ by the geometrical relations

$$
<x z>=\frac{R^{2}}{7} \frac{\omega_{z}^{2}-\omega_{\perp}^{2}}{\omega_{z} \omega_{\perp}} \theta \cos \phi, \quad<y z>=\frac{R^{2}}{7} \frac{\omega_{z}^{2}-\omega_{\perp}^{2}}{\omega_{z} \omega_{\perp}} \theta \sin \phi
$$

where $R=\sqrt{R_{\perp} R_{z}}$ and we have assumed $\theta \ll \epsilon$.

The crucial problem now is to obtain the equations for the averages $\langle x z\rangle$ and $\langle y z\rangle$ in the presence of the vortex. This problem was solved in 12 where it was shown that, in the Thomas-Fermi limit, the quadrupole operators $q_{ \pm}=\sum_{k=1}^{N}\left(x_{k} \pm i y_{k}\right) z_{k}$ excite, respectively, just one collective mode $\mid \pm>$ with frequency $\omega_{ \pm}$. The solution of the Schrödinger equation, in the presence of a small perturbation generated by these operators, then takes the form

$$
\mid \Psi(t)>=e^{-i E_{0} t / \hbar}\left[\left|0>+c_{+} e^{-i \omega_{+} t}\right|+>+c_{-} e^{-i \omega_{-} t} \mid->\right]
$$


where $\mid 0>$ is the equilibrium configuration, $\mid \pm>$ are eigenstates of the Hamiltonian corresponding to the elementary excitations created by $q_{ \pm}$and the complex parameters $c_{ \pm}$ characterize the initial state of the system. One can also show that the strengths of the quadrupole operators $q_{ \pm}$are equal: $<0\left|q_{-} q_{+}\right| 0>=<0\left|q_{+} q_{-}\right| 0>$, while the splitting between the two frequencies takes the simple form 12

$$
\omega_{+}-\omega_{-}=\frac{<l_{z}>}{m<x^{2}+z^{2}>}
$$

In eq.(7) the quantity $\left\langle\ell_{z}>\right.$ is the angular momentum per particle in the equilibrium configuration, while the average square radii can be easily evaluated in the Thomas-Fermi limit where one finds $<x^{2}>=<y^{2}>=(2 / 7) \mu / m \omega_{\perp}^{2}$ and $<z^{2}>=(2 / 7) \mu / m \omega_{z}^{2}$. Result (7) holds if the splitting is small compared to the unperturbed value (3) of the collective frequency.

The scenario emerging from the above discussion is quite clear. In the absence of vortices $\left(<l_{z}>=0\right)$ the two modes $\mid \pm>$ are degenerate and their frequency is given by (3). This is a simple consequence of time reversal symmetry. In the presence of the vortex there is a lift of degeneracy which is determined by the angular momentum of the system according to (7) [18]. By expressing the average values $\langle x z\rangle$ and $\langle y z\rangle$ in terms of the expectation values of the operators $q_{ \pm}$and using explicitly eq. (6), one derives the following time dependence for the angles $\theta$ and $\phi$ :

$$
\begin{gathered}
\theta(t) \cos \phi(t)=\alpha \cos \omega_{+} t+\alpha^{\prime} \sin \omega_{+} t+\beta \cos \omega_{-} t+\beta^{\prime} \sin \omega_{-} t \\
\theta(t) \sin \phi(t)=-\alpha^{\prime} \cos \omega_{+} t+\alpha \sin \omega_{+} t+\beta^{\prime} \cos \omega_{-} t-\beta \sin \omega_{-} t
\end{gathered}
$$

where the real coefficients $\alpha, \alpha^{\prime}, \beta$ and $\beta^{\prime}$ depend on the initial conditions for $\theta$ and $\phi$. For example the normal modes $\mid \pm>$ can be separately excited by choosing $\alpha=\theta_{0}, \alpha^{\prime}=\beta=$ $\beta^{\prime}=0$ and $\beta=\theta_{0}, \alpha=\alpha^{\prime}=\beta^{\prime}=0$ respectively. In both cases the inclination angle $\theta$ remains constant, $\theta(t)=\theta_{0}$, while the azimuthal angle $\phi$ precesses according to the laws $\omega_{+} t$ and $-\omega_{-} t$, respectively. 
In the most relevant case of a sudden rotation in the $x z$ plane, the initial conditions instead correspond to $\theta(0)=\theta_{0}, \theta^{\prime}(0)=\phi(0)=\phi^{\prime}(0)=0$. In this case the parameters of eqs.(89) are $\alpha=\beta=\theta_{0} / 2, \alpha^{\prime}=\beta^{\prime}=0$ and the solutions take the form

$$
\phi(t)=\frac{\left(\omega_{+}-\omega_{-}\right) t}{2}
$$

and

$$
\theta(t)=\theta_{0} \cos \left[\frac{\left(\omega_{+}+\omega_{-}\right) t}{2}\right]
$$

The simultaneous excitation of the $\mid \pm>$ modes is easily understood by noticing that the rotation of the trap produces a change in the external potential of the form $\delta V_{\text {ext }}=$ $m\left(\omega_{\perp}^{2}-\omega_{z}^{2}\right) \theta_{0} x z$. This gives rise to a perturbative term in the Hamiltonian, proportional to the combination $\left(q_{+}+q_{-}\right)$of the quadrupole operators introduced above. Equations (10) and (11) show that the scissors oscillation, characterized by the frequency $\left(\omega_{+}+\omega_{-}\right) / 2 \sim \omega_{\text {scissors }}$, undergoes a precession $d \phi / d t$ fixed by the splitting (7). The relative precession can be expressed in the form

$$
\frac{\omega_{+}-\omega_{-}}{\omega_{+}+\omega_{-}}=\frac{7}{2} \frac{<\ell_{z}>}{\hbar} \frac{\lambda^{5 / 3}}{\left(1+\lambda^{2}\right)^{3 / 2}}\left(15 N \frac{a}{a_{h o}}\right)^{-2 / 5}
$$

where $\lambda=\omega_{z} / \omega_{\perp}=\sqrt{(1-\epsilon) /(1+\epsilon)}$ and we have used the Thomas-Fermi result for $\left\langle x^{2}\right\rangle$ and $<z^{2}>$. The precession frequency $d \phi / d t$, and hence the ratio (12), depends explictly on the value of the angular momentum per particle which, in the case of a single quantized vortex aligned along the symmetry axis of the condensate, is given by $\left\langle\ell_{z}\right\rangle=\hbar$. The ratio (12) depends also on the shape of the trap. This provides further flexibility to optimize the visibility of the precession. In typical experimental configurations the Thomas-Fermi combination $\left(15 \mathrm{Na} / \mathrm{a}_{h o}\right)^{-2 / 5}$ is of the order of $10^{-2}$ so that for a highly elongated trap $(\lambda \ll 1)$ the relative precession is small. For values of $\lambda$ closer to unity the ratio (12) becomes larger. With suitable choices of the parameters of the trap the precession can be easily of the order of $1 \mathrm{~Hz}$ and should be consequently observable by imaging the atomic cloud at different times. 
In conclusion we have shown that Bose-Einstein condensed gases confined in harmonic traps can be used to realize a quantum gyroscope characterized by two important superfluid effects: the reduced value of the inertia of the sample and the quantization of the angular momentum associated with the vortex. The proposed gyroscope is characterized by the precession of the symmetry axis of the condensate around the symmetry axis of the confining trap, the precession frequency being fixed by the angular momentum carried by the vortex line. The experimental realization of the proposed gyroscope would provide a further tool to explore the intriguing features exhibited by rotating Bose-Einstein condensed gases, including the stability [19,20] and the life time [16] of vortex lines.

It is a pleasure to thank E. Cornell, J. Dalibard, A. Fetter, R. Packard, J. Reppy, E. Varauqaux and J. Vinen for useful comments and discussions. I also like to thank the hospitality of the Lorentz Center in Leiden and of the Newton Institute in Cambridge. This research is supported by the Ministero della Ricerca Scientifica e Tecnologica (MURST). 


\section{REFERENCES}

[1] M.H. Anderson et al., Science 269, (1995); C.C. Bradley et al, Phys. Rev. Lett. 75, 1687 (1995); K.B. Davis et al., Phys. Rev. Lett. 75, 3969 (1995);

[2] M.R. Matthews et al., Phys. Rev. Lett. 83, 2498 (1999).

[3] K.W. Madison et al., Phys. Rev. Lett. 84, 806 (2000).

[4] O.M. Marago' et al., Phys. Rev. Lett. 84, 2056 (2000).

[5] C. Raman et al., Phys. Rev. Lett. 83, 2502 (1999); A.P. Chikkatur, Phys. Rev. Lett. $85,483(2000)$.

[6] J.B. Mehel and W. Zimmermann, Jr., Phys. Rev. 167, 214 (1968); J.R. Clow and J.D. Reppy, Phys. Rev. A 5, 424 (1972).

[7] P.L. Gammel, H.E. Hall and J.D. Reppy, Phys. Rev. Lett. 52, 121 (1984); J.P. Pekola et al., Phys. Rev. Lett., 53, 70 (1984).

[8] F. Dalfovo, S. Giorgini, L. Pitaevskii and S. Stringari, Rev. Mod. Phys. 71, 463 (1999).

[9] F. Chevy, K.W. Madison and J. Dalibard, Phys. Rev. Lett. 85, 2223 (2000).

[10] B.P. Anderson, P.C. Haljan, C.E. Wieman and E.A. Cornell, Phys. Rev. Lett. 85, 2857 $(2000)$.

[11] P.C. Haljan, B.P. Anderson, I. Coddington, E.A. Cornell, cond-mat/0012320.

[12] F. Zambelli and S. Stringari, Phys. Rev. Lett. 81, 1754 (1998).

[13] S. Sinha, Phys. Rev. A 55, 4325 (1997); R. Dodd et al., Phys. Rev. A 56, 587 (1997);

A. Svidzinsky and A. Fetter, Phys. rev. A 58, 3168 (1998).

[14] D. Guery-Odelin and S. Stringari, Phys. Rev. Lett. 83, 4452 (1999).

[15] D. Rokhsar, Phys. Rev. Lett. 79, 2164 (1997). 
[16] P.O. Fedichev and G.V. Shlyapnikov, Phys. Rev. A 60, R1779 (1999).

[17] Ph. Nozieres and D. Pines, The theory of quantum liquids (Addison-Wesley, Reading, MA, 1990), Vol. II; S. Stringari, Phys. Rev. Lett. 77, 2360 (1996);

[18] A similar scenario takes place if we excite the system with the quadrupole operators $\sum_{k=1}^{N}\left(x_{k} \pm i y_{k}\right)^{2}$. This choice corresponds to generating a quadrupole deformation in the plane perpendicular the symmetry axis, like in the experiment of [9]. In this case the unperturbed frequency of the quadrupole oscillation is $\sqrt{2} \omega_{\perp}$, while the splitting takes the form $\omega_{+}-\omega_{-}=(2 / m)<\ell_{z}>/\left(<x^{2}>+<y^{2}>\right)$ [12.

[19] A. Svidzinsky and A. Fetter, Phys. Rev. Lett. 845919 (2000) and e-print condmat/0007139; see also A. Svidzinsky and A. Fetter, J. Phys. G, in press, and references therein.

[20] J.J. Garcia-Ripoll and V.M. Perez-Garcia, cond-mat/0012071.

\section{FIGURE CAPTION:}

Schematic picture of the BEC gyroscope. The condensate is suddenly rotated with respect to the symmetry axis of the trap ( $z$-th axis) and exhibits precession in the presence of a quantized vortex line. 


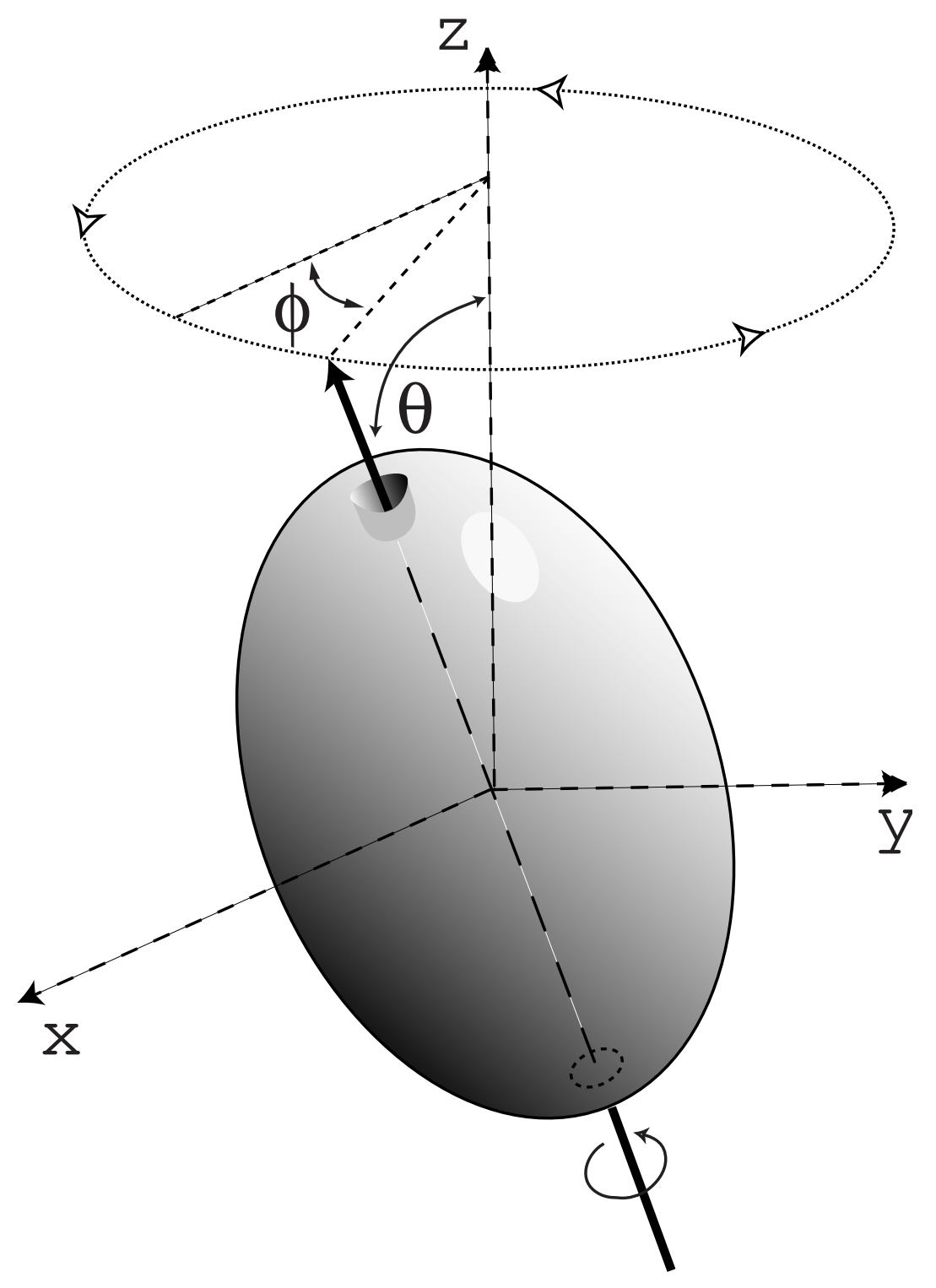

